\title{
All-Solid-State Ion-Selective Electrodes: A Tutorial for Correct Practice
}

\author{
Francesca Criscuolo ${ }^{(}{ }^{-}$, Mandresy Ivan Ny Hanitra ${ }^{\circledR}$, Member, IEEE, Irene Taurino, \\ Sandro Carrara ${ }^{\circledR}$, Fellow, IEEE, and Giovanni De Micheli ${ }^{\circledR}$, Life Fellow, IEEE
}

\begin{abstract}
The research on ion-measuring devices has seen a rapid expansion in the last years as a consequence of the growing interest in wearable sensors for biofluids analysis and in portable devices for remote or in-line water and food quality monitoring. As a result, an increasing number of researchers is approaching the field of ion-selective sensors. Despite the apparently simple transduction principle, the the-

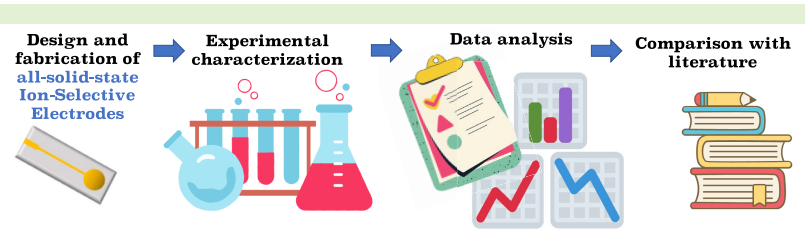
ory behind their working mechanism is far from trivial and a correct understanding is necessary for an optimal exploitation of the technology. In current literature, imprecise characterization procedures lead to the definition of misleading sensors parameters, which cannot be effectively used for comparisons. In fact, some unique definitions and procedures applies to this category of sensors, which significantly differ from the traditional ones applied in sensing research. This tutorial aims at highlighting the basic thermodynamic theory and the correct experimental practice for the accurate and reproducible characterization of potentiometric ion-sensors. The most important requirements and design considerations on hardware and software interfaces will also be discussed to give a complete overview of the various aspects of current technology to people approaching this promising area of sensing.
\end{abstract}

Index Terms-Ion-sensing, ion-selective electrodes, solid-contact, theory, practice, guidelines, sensor parameters, state-of-the-art.

\section{INTRODUCTION}

$\mathbf{T}$ HE research on ion-sensing is expanding rapidly in various fields, from medicine, to food and water quality monitoring, as well as control of industrial production. Currently, the most used sensors for ion-quantification are Ion-Selective Electrodes (ISEs), electrochemical systems that convert the activity of a target ion into an electrical potential as the measurable signal. The need for miniaturized system for wearable or on-line applications brought the technology to the development of all-solid-state ISEs. These devices enable smaller and accurate sensing, with characteristics suitable for conformal or portable devices [1]-[5].

The most investigated application area is certainly healthcare. In fact, electrolyte balance provides significant information on patient's health [6]. The first and so-far most studied fields are the monitoring of hydration and physical exercise $\left(\mathrm{Na}^{+}\right.$and $\left.\mathrm{K}^{+}\right)$[7], [8], and the diagnosis of cystic

Manuscript received May 21, 2021; revised July 19, 2021; accepted July 20, 2021. Date of publication July 26, 2021; date of current version October 18, 2021. This work was supported by H2020 CyberCare under Grant ERC 2014 ADG669354. The associate editor coordinating the review of this article and approving it for publication was Dr. Marios Sophocleous. (Francesca Criscuolo and Mandresy Ivan Ny Hanitra contributed equally to this work.) (Corresponding author: Mandresy Ivan Ny Hanitra.)

The authors are with the Laboratory of Integrated System, EPFL, 1015 Lausanne, Switzerland (e-mail: ivan.nyhanitra @epfl.ch).

Digital Object Identifier 10.1109/JSEN.2021.3099209 fibrosis $\left(\mathrm{Cl}^{-}\right)$[9]. However, research is rapidly expanding towards other sectors of medicine as well, including the control of heavy metal contamination in people exposed to risky environments $\left(\mathrm{Pb}^{2+}, \mathrm{As}^{2+}\right)[10]$, the control of liver and bones status $\left(\mathrm{NH}_{4}^{+}\right.$and $\mathrm{Ca}^{2+}$, respectively) [11], the therapeutic drug monitoring in people suffering from bipolar disorder $\left(\mathrm{Li}^{+}\right)$[12], as well as skeletal and dental studies $\left(\mathrm{F}^{-}\right)$.

Apart from medical analysis, ion-quantification is fundamental also for other relevant applications, like agriculture, fishery, food and water quality monitoring, industrial control. Most of the times these applications involve water solutions, thus interference is expected to be much lower than in bodily fluids, thus facilitating the sensing. In particular, considerable attention has been attracted by water quality monitoring [13]-[16]. We recommend this tutorial for more details on this specific topic [17]. Possible applications of ion-sensors outside medical care also include: control of salt content in food; fluoride content in drinking water and drinks, calcium monitoring in dairy products and beer, potassium in fruit juices and wine making; agriculture $\left(\mathrm{Na}^{+}, \mathrm{K}^{+} \mathrm{NO}_{3}, \mathrm{Cl}^{-}, \mathrm{NH}_{4}\right.$ in soils, plant material and fertilisers); pollution-monitoring in effluents and natural waters $\left(\mathrm{F}^{-}, \mathrm{S}^{-2}, \mathrm{Cl}^{-}, \mathrm{NO}_{3}\right)$; study of the effect of detergent manufacture on water quality $\left(\mathrm{Ca}^{2+}\right.$, $\left.\mathrm{Ba}^{2+}, \mathrm{F}^{-}\right)$; control of electroplating process $\left(\mathrm{F}^{-}, \mathrm{Cl}^{-}\right.$and $\mathrm{S}^{-2}$ ) [18], [19].

It is clear that ion-sensing occupies a primary role in current sensing research. So far, the most efficient and precise ion- 
sensing devices employ electrochemical measurements using ISEs [20]. Despite this rapid growth, there is still large confusion in the scientific community about the theory and correct practice behind their characterization and use, as some unique definitions and protocols apply to this category of sensors. As a prime example, the traditional definition of Limit-OfDetection (LOD) does not apply for this type of sensors, as a more restrictive one is more appropriate and recommended by the International Union of Pure and Applied Chemistry (IUPAC). In addition, precise conditioning protocols must be applied to obtain accurate and comparable values. These peculiar characteristics of ISEs are often confused and ignored by many experts in the field of sensing that approach ions for the first time.

The aim of this review is to highlight the correct definitions and practice of this unique branch of sensing, in order to avoid inaccuracy in the determination of sensor parameters that causes misleading and incomparable values among different research groups. In Section II, a brief summary of the most important thermodynamic parameters necessary for the correct use of these sensors is provided. This is followed by the description of the working principle of conventional ISEs and their evolution towards all-solid-state systems for miniaturized and portable applications are reported. In Section III, the procedure for the accurate characterization of solid-contact ISEs is detailed, with particular emphasis on common misunderstandings. Section IV gives an overview of hardware and software interfaces for these types of sensors, stressing the most suitable approaches and design considerations.

$\begin{array}{ll}\text { A. Abbreviations and Acronyms } \\ \text { ADC } & \text { Analog-to-Digital Converter } \\ \text { CMRR } & \text { Common-Mode Rejection Ratio } \\ \text { CRC } & \text { Current Reversal Chronopotentiometry } \\ \text { DC } & \text { Direct Current } \\ \text { FIM } & \text { Fixed Interference Method } \\ \text { ISE } & \text { Ion-Selective Electrode } \\ \text { ISM } & \text { Ion-Selective Membrane } \\ \text { IUPAC } & \text { International Union of Pure and Applied } \\ & \text { Chemistry } \\ \text { LOC } & \text { Lab-on-Chip } \\ \text { LOD } & \text { Limit of Detection } \\ \text { MCU } & \text { Micro-Controller Unit } \\ \text { MPM } & \text { Matched Potential Method } \\ \text { OCP } & \text { Open Circuit Potential } \\ \text { PCB } & \text { Printed Circuit Board } \\ \text { POC } & \text { Point-of-Care } \\ \text { RE } & \text { Reference Electrode } \\ \text { SC } & \text { Solid Contact } \\ \text { SC-ISE } & \text { Solid-Contact Ion-Selective Electrode } \\ \text { SSM } & \text { Separate Solution Method } \\ \text { WE } & \text { Working Electrode }\end{array}$

II. ION-Selective Electrodes (ISEs) THEORY AND WORKING PRINCIPLES

ISEs are potentiometric sensors based on the deposition on the sensor surface of an Ion-Selective Membrane (ISM) that contains a species, called the ionophore, that selectively binds to the target ion. Larger ion concentrations in solution causes the trapping of higher amounts of ions in the ISM. This results in a measurable and proportional Open Circuit Potential (OCP) with respect to a stable Reference Electrode (RE).

These devices offer several advantages over other technologies, including the relative simplicity of OCP measurements, their low cost and maintenance. A wide concentration range can be measured with these systems, making them very flexible to the user's needs. Both cations and anions can be detected in a broad temperature window. For these reasons, they are ideal candidates for a variety of applications.

The direct measurement of ion activity, rather than its concentration, is particularly useful in biological and medical analysis. With careful use, accuracy and precision levels of 2-3\% can be achieved for some ions. This performance is comparable to the most specialized analytical techniques, which require complex and expensive instrumentation [20]. In addition, it is possible to mass produce arrays of miniaturized sensors for simultaneous analysis of cations and anions mixtures, with possibilities of simple and compact integration [7], [21]. Moreover, the measurements do not require high voltages as in capillary electrophoresis. Finally, the direct correlation between the measured potential and ion activity suggests the absence of a scaling law and of signal reduction with reduced electrode dimensions [21]-[23].

Despite the wide success of ISEs, other types of sensors have also been investigated for ion-sensing [24], including luminescent [18] and colorimetric [25], [26] detection. However, such optical sensors necessitate more elaborated and larger system implementations, thus they are not suitable for miniaturized on-line sensing systems that are the main focus of current ion research.

In the next subsections, the main thermodynamic concepts in ion-sensing are defined, along with the working mechanism of conventional ISEs. Then, the evolution towards all-solidstate ISEs is described, emphasizing its importance for sensor miniaturization.

\section{A. Ion Activity and Activity Coefficients}

The understanding of some basic thermodynamic concepts is essential for a thorough grasp of the theory and correct definition of the most important analytical parameters of ISEs. In this subsection, an introduction of the main thermodynamic notions needed in ion sensing is provided. In particular, the activity of an analyte, and its single ion activity coefficient are carefully distinguished. Furthermore, we define the mean activity coefficient and the ionic strength of a solution.

The activity describes the effective concentration of a solution. The equilibrium in ionic solutions depends on ion activities. The activity of an ion $a_{j}$ in solution is proportional to its molar concentration $c_{j}$ according to the following equation [27]:

$$
a_{j}=\gamma_{j} c_{j},
$$

where $\gamma$ is the single ion activity coefficient. Its value cannot be obtained experimentally. Nonetheless, in most practical cases, the single ion activity coefficient for anions $\gamma_{-}$, 
or cations $\gamma_{+}$, can be calculated from the mean activity coefficient of the solution $\gamma_{ \pm}$, which is defined as:

$$
\log \gamma_{ \pm}=\frac{\left|z_{-}\right|}{\left|z_{+}\right|+\left|z_{-}\right|} \log \gamma_{+}+\frac{\left|z_{+}\right|}{\left|z_{+}\right|+\left|z_{-}\right|} \log \gamma_{-},
$$

where $z_{-}$and $z_{+}$are the valences of the anions and cations, respectively. The splitting of the mean activity coefficient into the relevant single ion activity coefficients can be performed with different conventions. The most precise calculation is the one obtained by using the hydration theory of Stokes, Robinson and Bates. However, in ordinary analytical procedure, the simplified Debye-Hückel convention [27] is sufficiently accurate:

$$
\begin{aligned}
& \log \gamma_{+}=\left|\frac{z_{+}}{z_{-}}\right| \log \gamma_{ \pm} \\
& \log \gamma_{-}=\left|\frac{z_{-}}{z_{+}}\right| \log \gamma_{ \pm}
\end{aligned}
$$

The extended Debye-Hückel equation can be used in most cases to evaluate the mean activity coefficient $\gamma_{ \pm}$[27]:

$$
\log \gamma_{ \pm}=-\frac{A\left|z_{+} z_{-}\right| \sqrt{I}}{1+B \sqrt{I}}+C I .
$$

This is a semi-empirical relationship. A is a parameter that varies with temperature in Kelvin as $T^{-3 / 2}$. For standard condition $\left(\mathrm{T}=25^{\circ} \mathrm{C}\right)$ in water solutions, $\mathrm{A}=0.5108$. $\mathrm{B}$ and $\mathrm{C}$ are thermodynamic parameters depending on the electrolyte. A list of their experimental values is reported in [28]. I is the ionic strength of the solution, which by definition is given by:

$$
I=0.5 \sum_{j} z_{j}^{2} c_{j} .
$$

\section{B. Structure and Working Mechanism of Conventional ISES}

The structure and working mechanism of a conventional ISE is shown in Figure 1a and 1b. The measurement is performed in a two-electrodes setup. Conventional systems are characterized by the presence of an internal liquid junction (i.e. of an internal filling solution at constant concentration) at the Working Electrode (WE) to maximize stability. This clearly makes these systems macroscopic. The ISM of the WE is used to separate the internal liquid junction from the sample solution. The ISM allows the flow and entrapment only of the primary ion, thanks to the presence of a neutral chemical compound called the ionophore, which selectively binds to the target ion $\mathrm{M}^{+}$.

In order to maximize sensing performance, the recommended ISM cocktail should in general include 1-2\% of ionophore, $60-70 \%$ of plasticizer, and 30-40\% of high molecular weight PolyVinyl Chloride (PVC). The plasticizer increases the free volume of the polymeric materials, thus enhancing flexibility, mobility, and durability. Typically-used plasticizers are bis(2-ethylhexyl)sebacate (DOS) or ortho-nitrophenyl octyl ether (o-NPOE). The polymeric matrix provides mechanical stability and is chemically inert. Small quantities of lipophilic ions are added to the solution to avoid the removal of large amounts of counterions from the sample solution to

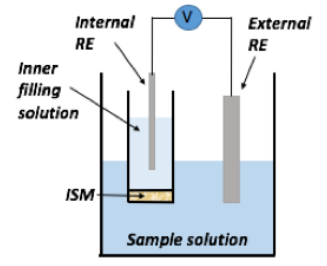

(a)

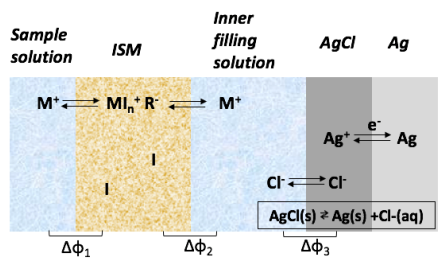

(b)

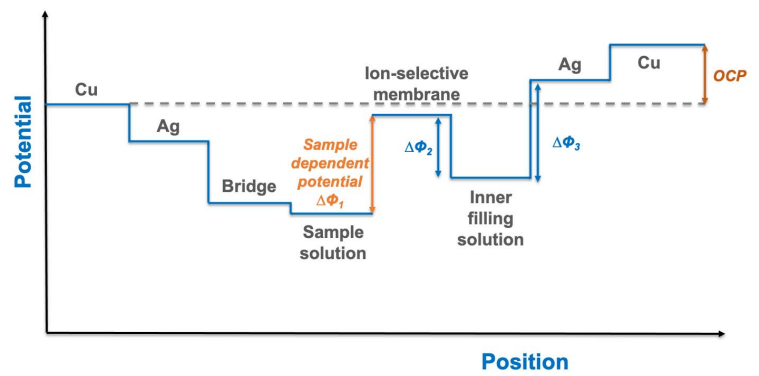

(c)
Fig. 1. (a) Structure and working principle of conventional ISEs (i.e. with inner filling solution). (b) Electrochemical setup used in the potentiometric measurements with ISEs. (c) Potential profile within a cell containing a conventional ISE and a liquid junction RE. The RE bridge consists of a reference electrolyte solution separated from the sample solution by a porous frit. The sample dependent potential is the one of interest for the sensor calibration.

the ISM. In fact, this method makes the membrane permeable exclusively to ions of the same sign of the target ion. This is called Donnan exclusion and is necessary to achieve ideal sensor response. Tetraphenylborates are commonly employed for this purpose in cation-selective ISM, whereas tetraalkylammonium salts are often used in anion-selective membranes [6], [20], [29].

Whenever the concentrations of the ions in the inner and the sample solution are not equal, a potential difference is built across the membrane as a consequence of ion exchange and trapping in the ISM. By measuring the OCP of the ISE with respect to a RE with stable potential, we can predict the activity, i.e. the concentration, of the target ion in solution. In particular, by keeping constant the activity of the target ion in the internal solution $a_{A}$, it is possible to determine the unknown activity on the other side of the membrane $a_{x}$, using Nernst equation which defines the electrode potential $\mathrm{E}$ as:

$$
E=\frac{R T}{z_{A} F} \ln \left(\frac{a_{x}}{a_{A}}\right)=\text { const }+2.3 \frac{R T}{z_{A} F} \log a_{x},
$$

where $R$ is the gas constant, $T$ the temperature, $z_{A}$ the valence of the target ion, and $F$ Faraday's constant. The internal liquid junction acts as a liquid contact enabling ion-toelectron transduction by exploiting the reaction $\mathrm{AgCl}(s) \rightarrow$ $\mathrm{Ag}(s)+\mathrm{Cl}^{-}(a q)$. This defines the interfacial potential $\Delta \phi_{3}$. It is worth to underline that ISEs can detect free, not complex, ion concentrations [20], [22], [30], [31].

ISEs are typically measured in a two-electrodes setup (Figure 1a), where the ISE acts as the cathode, and the RE as the anode. The measured potential difference is given by the sum of all potential differences in the cell (Figure 1c). Therefore, it is clear that all potentials must be kept 


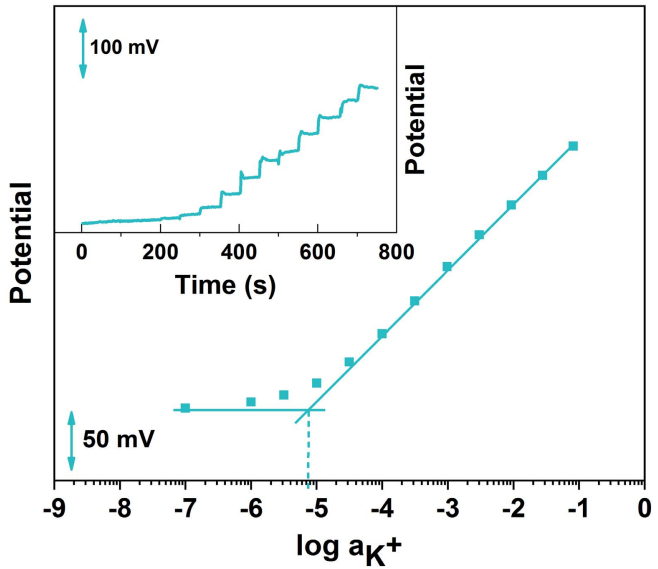

Fig. 2. Example of calibration curve obtained with potentiometric $\mathrm{K}^{+}$ ISES.

independent of sample solution, expect the one at the interface sample/membrane (in orange) [20].

By definition, the calibration curve of a sensor is the plot of the output signal as a function of the analyte concentration. Observing Nernst equation (7), we can notice that in principle, potentiometric ISEs offer a linear relationship when the potential is plotted with respect to the log of the ion concentration. A typical calibration curve for this type of sensors is given in Figure 2. The slope of the calibration curve, that is $\frac{R T}{z_{A} F}$, is the sensitivity of the sensor $S . \frac{R T}{F}$ is equal to $59.16 \mathrm{mV}$ at room temperature. The sensitivity of potentiometric sensors depends mainly on the target ion charge: monovalent cations show an ideal calibration slope of $59 \mathrm{mV} /$ decade, which means that a 10-fold concentration will generate a change of $59 \mathrm{mV}$ in the measured potential; divalent cations will have a calibration curve with a slope of $29.5 \mathrm{mV} /$ decade. The same reasoning can be applied to anions, but in this case negative sensitivity will be observed.

\section{All-Solid-State ISES}

As explained in the previous sections, conventional ISEs are based on an internal liquid junction, that is, an internal filling solution at constant concentration, allowing a minimization of potential drifts. Conventional systems are very accurate, with high stability and long lifetime [20], [22]. However, the internal electrolyte of ISEs is prone to evaporation and highly sensitive to temperature and pressure variations, that can lead to large volume changes and eventually to delamination. Moreover, and most importantly, the filling solution limits the possibility of miniaturizing these systems. This prevents their applicability for Lab-On-a-Chip (LOC), wearable, or online devices, where sensor dimension is a paramount factor. To overcome this major drawback, all-solid-state potentiometric ion-sensors have been developed over the past 50 years towards next generation portable and miniaturized ion-sensing devices, with integration of steering circuits and read-out electronics [20]. These ISEs exploit Solid-Contacts (SCs) between the ISM and the electronic conductive substrate to achieve ion-to-electron transduction. For this reason, they are also called Solid-Contact ISEs (SC ISEs). Their fabrication is based on the deposition of a properly designed ISM on top of a metal electrode to attract selectively the target ions close to the surface; the change in charge distribution due to the accumulation of ions at the electrode surface is detected by simply measuring the OCP with respect to the RE. Different ions-to-electron transduction mechanisms can be exploited [20], [22], [30], [31].

The first all-solid-state ISE was proposed in 1970 by Hirata and Date [32], followed by another one proposed by Cattrall et al. [33] the year after. Both systems were based on coated-wire electrodes. Poor reliability was achieved mainly because of the purely capacitive interface, and because of the reduced interfacial contact area. Since then, several advances have been made thanks to the use of new SC materials, and a better understanding of transport phenomena and water accumulation in the membrane. In particular, the most promising candidates for commercial SC ISEs are conductive polymers and nanostructures with high double layer capacitance. These two classes of materials exploit different ion-to-electron transduction mechanisms [6], [34]-[36]. The next two subsections describes their working principle and main characteristics.

\section{Solid-Contacts Based on Conductive Polymers.}

One of the most investigated SC materials for ISEs are conductive polymers, as they are able to conduct both ions and electrons when properly doped. For this reason, they can be exploited as effective ion-to-electron transducers through redox reactions (Figure 3a). Polypyrrole [37], polyaniline [38], poly(3-octyltiophene) (POT) [39] and poly(3,4-ethylenedioxytiophene (PEDOT) [40], are nowadays the most investigated conductive polymers in SC ISEs. They can be deposited by electropolymerization or drop casting from polymeric solutions. Polyaniline, polypyrrole and PEDOT show high stability, conductivity and redox capacitance. However, they are electroactive in a wide range of potentials. This can cause several side reactions and consequent potential drift. Furthermore, the redox potential can vary significantly with the crystallinity degree, film morphology and doping effect on the glass transition temperature. Conversely, POT participates much less in side reactions, but presents a much lower redox capacitance and conductivity [20], [30].

Solid Contacts Based on Nanostructured Layers:

Nanostructured SCs are based on the formation of an electrical double layer at the membrane/electrode interface for ion-to-electron transduction: the trapping of ions on one side of the interface thanks to the role of the ISM provokes the accumulation of electrons and holes on the other side; this causes the generation of an asymmetric capacitor. In such systems, the interfacial potential is not due to redox reactions, as in the case of conductive polymers, or to ion partitioning, as for conventional ISEs, but to the amount of charge accumulated in the double layer. This leads to the build-up of the potential difference $\Delta \Phi_{2}$ (Figure 3b). The large surface area facilitates adhesion, avoiding the risk of water absorption, while the high capacitance values reduces potential drift due to polarization effects caused by the small but non-zero currents required for the measurement. Both carbon and noble metals nanostructures have been successfully investigated in the research community for SC ISEs [20], [29], [41]-[48]. 


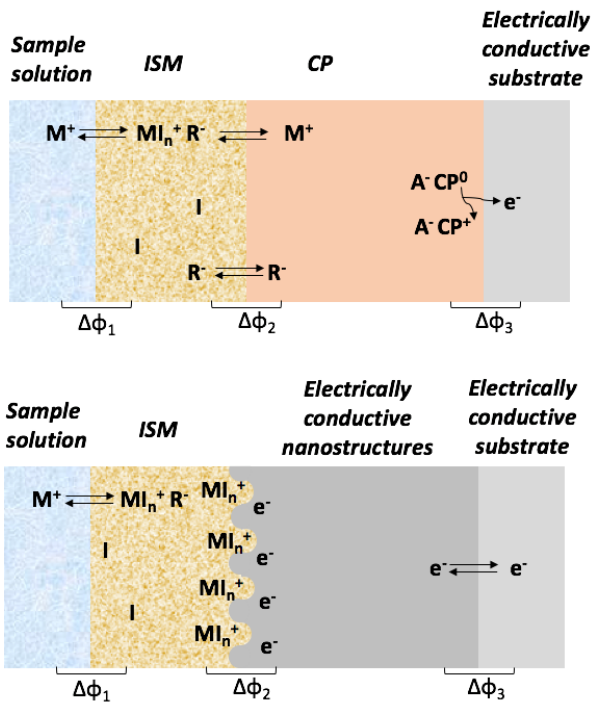

Fig. 3. a) Scheme and working mechanism of ISEs with SCs based on conductive polymers (CP). b) Scheme and working mechanism of ISEs with SCs based on nanostructured SCs. I represents the ionophore, $M$ the metal, $R$ is an anionic exchanger.

\section{Performance Evaluation Criteria of ISES}

In order to properly characterize and compare sensors performance, it is crucial to define meaningful and reproducible sensing evaluation criteria. It is clear that the main requirements for the design and fabrication of a sensor will depend on the specific application, but in general, the most important parameters in every sensing system are the following:

- Sensitivity - absolute amount of change that can be detected by a measurement;

- Limit-Of-Detection (LOD) - minimum analyte concentration that can be reliably sensed;

- Dynamic linearity range - ability to follow linearly variations of analyte concentration in a certain concentration range;

- Selectivity - ability to determine the concentration of a substance in the presence of interfering substances.

Furthermore, the use of SC ISEs in portable, wearable and on-line sensing systems requires that several other characteristics are met: reproducibility, precision, biocompatibility, stability over time in order to monitor patients for several hours (and eventually even longer) without calibration. Among these properties, potential drift represents certainly one of the most critical issues of ISEs. For this reason, it is important to perform appropriate characterization of potential stability. In the next subsections, we will describe more precisely the most significant sensor parameters, with particular focus on the correct practical procedure to avoid incurring inaccurate or incomparable values.

\section{A. Sensitivity}

Sensors calibration is clearly the most fundamental procedure to characterize sensor analytical performance. In order to calibrate a sensor, its response (e.g. OCP for ISEs) is recorded at increasing and known concentrations of target analyte. The potential value at each concentration step is taken to build a calibration curve showing the relationship between the sensor output and the concentration of the target analyte. The slope of the calibration plot is called sensor sensitivity and is an essential characterization parameter of any sensor [6]. A typical experimental calibration curve is reported in Figure 1c, where the inset shows the time trace at increasing concentration from which the calibration graph was calculated.

As explained before, a linear relation is usually found when the OCP is plotted against the logarithm in base 10 of the target ion activity, as stated by Nernst equation (7). At ambient temperature, an ideal ISE exhibits a Nernstian slope of $59.2 \mathrm{mV} /$ decade for monovalent ions, and of $29.5 \mathrm{mV} /$ decade for divalent ions. However, in reality these values are not always attained. In order to achieve an ideal behaviour, the membrane must be perfectly selective to the target ion and the RE potential must be independent of sample composition. In addition, the membrane-target ion binding must be fully reversible, and the membrane composition must remain constant over time. In practice, some non-idealities are often present. For instance, in AgCl-based membranes, the surface is frequently modified by other less soluble halides [20], [22], [30], [31].

\section{B. Limit-Of-Detection (LOD)}

The definition of LOD for ISE is one of the most critical and often misleading aspects of their characterization, as a unique definition is applied for this class of sensors with respect to the traditional one. LOD is one of the most important parameters to characterize the analytical performance of a sensing device because it defines the application limit of the proposed technology. This is used to give an indication of the smallest concentration of analyte that can be reliably detected. In general, in analytical chemistry, the LOD is defined according to the IUPAC standard, as:

$$
L O D=\frac{3 \sigma_{b}}{\text { Sensitivity }},
$$

where $\sigma_{b}$ is the standard deviation of the signal of the blank (that is, a solution identical to the analyzed one, but without target analyte). The factor 3 maintains the minimum required confidence level of $99 \%$. In other words, the LOD is the concentration at which there is an increase of the signal with respect to the background level of the noise determined, as three times the standard deviation of the blank measurement [49].

However, the standard IUPAC definition for potentiometric ISEs is different from the traditional one. This issue is often ignored by many experts in the field of sensing who do not directly work with ISEs, or who approach this field for the first time. A deviation from the linear response is usually present in the calibration curve of ISEs at high and low activity concentrations. IUPAC recommends that the upper and lower LODs for ISEs are determined as the intercepts between different linear segments of the calibration curve, as shown in Figure 4. The upper LOD is caused by loss of permselectivity (also called Donnan Exclusion), that is the permeability only to ions with the same charge sign of the target ion. An ideal 


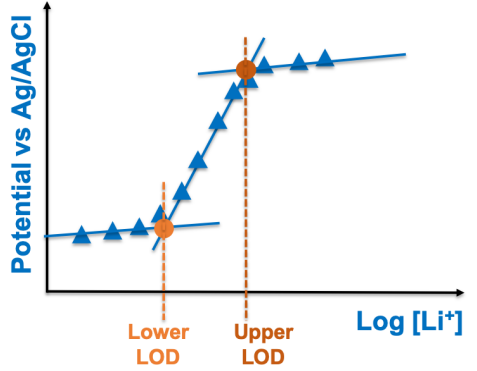

Fig. 4. Determination of upper and lower LOD according to IUPAC definition.

slope is observed only if no significant amount of counter ions (ions with opposite charge sign of the measuring ion) are coextracted together with the target ions from the sample into the membrane phase. Conversely, if ions of opposite sign are also attracted, we will observe a reduction of the measured OCP with respect to the expected value. The lower detection limit can be explained considering interference effects or leaching out of target ions from the membrane.

It is important to notice that the unique definition of LOD in potentiometric ISEs is more restrictive than the classical one. In fact, for an ideal membrane, the LOD corresponds to the situation in which a well-defined part $(50 \%$ for ions of the same charge) of the interfering ions replaces the target ones in the organic phase. This will give a deviation of $17.8 / z \mathrm{mV}$ in the potential [49], where $\mathrm{z}$ is the ion charge number. On the contrary, the typical definition of LOD, that is three times the standard deviation of the noise, would result in a much lower value, as in general the noise level in potentiometry is about $0.06-0.08 m V$ [6], [49].

It is worth mentioning that proper membrane conditioning is needed in order to make the membrane sensitive in a specific concentration range [6], [49] as this most of the times influences the low and upper LOD.

\section{Potential Response and Stability}

One of the most critical challenges in solid-contact ISEs is certainly represented by potential stability. It is clear then why the correct characterization of this aspect is essential for comparison purposes. A first step is the measurement of the sensor potential stabilization time when it comes in contact with a solution for the first time. In this regards, the selection of the electrically conductive substrate plays an important role [20], [50].

However, the most crucial issues of all-solid-state ISEs is represented by potential drift over a long period of time, that is potential instability. One of the most common and simple explanation related to this shortcoming is the formation of a water layer at the ISM/SC interface. This phenomenon can be deducted from various aspects of sensor performance: slow stabilization time, sensitivity to osmolality variations, positive potential drift upon addition of interfering ions, negative drift upon removal. The most commonly-used method for characterization of potential stability due to formation of an aqueous layer is the so-called water layer test [6]. This is based on the measurements of the potential during successive

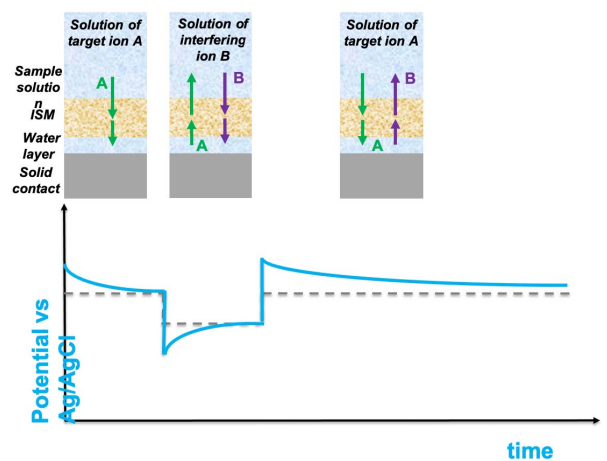

Fig. 5. Ion fluxes and typical potential profile during a water layer test for evaluating ISEs potential drift.

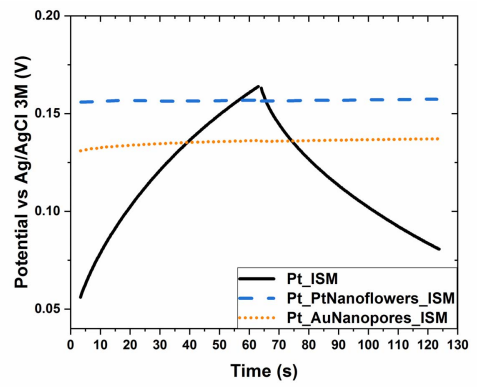

Fig. 6. CRC curves with and without nanostructured SCs. The response of the SC with gold and platinum nanostructures is compared. CRC are useful to determine the stability of the SC-ISE from potential drift over time. It is clear that the nanostructures in this case offer a significant improvement in potential drift (i.e. flatter curves).

exposures of the sensor to concentrated solutions (e.g. 0.1 M) of target and interfering ions for several hours. Even small ionic fluxes during ion exchanges can lead to large changes of the very thin water layer at the ISE/SC interface, leading to positive and negative drifts during exposure to the interfering and target ion, respectively. The mechanism of ion diffusion and consequent potential drifts is depicted in Figure 5.

Another very common method to characterize potential stability of ISEs is Current Reversal Chronopotentiometry (CRC). In this method, a direct current of a few nanoamperes is applied, and reversed after a certain period of time. The potential drift $\frac{\mathrm{dE}}{\mathrm{dt}}$ can be computed from the slope of the graph [6], [34]. A typical CRC curve is given in Figure 6 for a $\mathrm{Li}^{+}$ISE based on two different types of nanostructures, in comparison with the one deposited on a bare electrode (the solid black curve in the graph). The beneficial effect of electrode nanostructuration on potential stability is evident in both cases as the presence of this nanostructured SC enables the achievement of flat CRC curves(i.e. very small potential drift). On the contrary, the electrode without nanostructuration shows a major potential instability, as evident from the graph slope.

CRC is also useful to compute the electrode capacitance as $C=\frac{i}{d E / d t}$, where $i$ is the applied current, and $d E / d t$ is the potential drift [20]. A higher capacitance will in general result in a larger potential stability.

The formation of a water layer can be a consequence of water uptake during the fabrication process, even before the contact with an aqueous solution. For instance, Tetrahydrofu- 
ran (THF) is known to be highly hygroscopic. Freshly distilled THF and dry conditions can be employed in order to minimize this phenomenon, if needed [6], [29]. As explained before, potential drifts are not necessarily related to a water layer. It can also be caused by the small but non-zero currents required for the potential read-out. For this reason, it is important to assess the effect of current polarization on sensor response, especially on miniaturized electrodes ( $\mathrm{mm}$ or sub$\mathrm{mm}$ size) [6], [20]. Another possible source of potential instability is the progressive degradation of membrane composition. This can be related to the leaching out of ionophores or lipophilic salts from the ISM. This phenomenon can also be observed with CRC measurements [6], [20], [29].

\section{Interference Studies}

The selectivity of a sensor represents its ability to detect the concentration of the target substance in presence of interfering compounds. This parameter is essential to manufacture an efficient sensing system. During ion-sensing through an ISE, ions of identical charge sign as the target ion might be able to replace the target ions from the ISM, thus giving spurious signal. As explained in IUPAC reports [34], Nikolsky-Eisenman equation can be used to define the measured potential under the presence of interfering ions:

$$
E=E_{0}+\frac{R T}{z F} \ln \left[a_{A}+\sum_{B} K_{A, B}^{p o t}\left(a_{B}\right)^{\frac{z_{A}}{z_{B}}}\right],
$$

where $E_{0}$ is a constant including the standard potential of the electrode, $a_{A}$ and $a_{B}$ are the activities of the target and interfering ions respectively, $z_{A}$ and $z_{B}$ their valence, $K_{A, B}^{\text {pot }}$ the potentiometric selectivity coefficient of the target ion $A$ against the interfering ion B. The smaller the value of $K_{A, B}^{p o t}$, the higher is the selectivity of the sensor. If $K_{A, B}^{\text {pot }}<1$ the sensor is more selective towards the target ion. On the contrary, if $K_{A, B}^{\text {pot }}>1$, the sensor is more selective towards the interfering ions (this case is seldom). It is worth reminding that (9) can be used only when the following assumptions are satisfied: 1) the sensor exhibits a Nernstian response towards both ions; 2) the selectivity coefficient $K_{A, B}^{\text {pot }}$ does not vary; 3) target and interfering ions have the same charge (when mono and divalent ions are present, more complex equations must be used) [27], [34]. The selectivity coefficient in (9) acts as a weighting factor for any ion activity. An example of calibration graphs for membranes with different selectivity coefficients is reported in Figure 7. It is clear that the higher the value of $K_{A, B}^{\text {pot }}$, the larger the interference is, which in turn will result in a lower LOD. On the contrary, the sensitivity is in principle not affected.

According to IUPAC recommendations, three different methods are possible for the determination of the selectivity coefficients $K_{A, B}^{\text {pot }}$ [51]:

- the Separate Solution Method (SSM) I is based on the use of two different solutions for the measurements. The first one contains only target ions $\mathrm{A}$ at activity $a_{A}$, the second one has only interfering ions $\mathrm{B}$ at activity $a_{B}=a_{A}$.

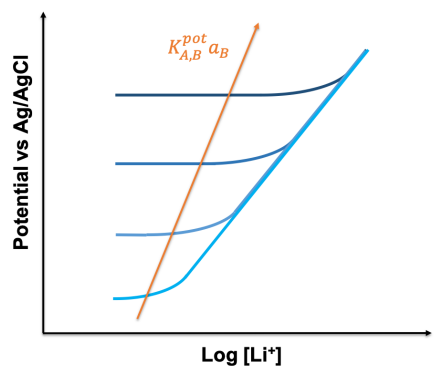

Fig. 7. Calibration curves of ISE with different selectivity coefficients $K_{A, B}^{p o t}$. The intersection of the two linear portions is the LOD. It is evident that a smaller selectivity coefficient $K_{A, B}^{\text {pot }}$ results in lower LOD, thus lower interference.

The selectivity coefficient is then calculated as:

$$
\log K_{A, B}^{p o t}=\frac{\left(E_{B}-E_{A}\right) z_{A} F}{2.303 R T}+\left(1-\frac{z_{A}}{z_{B}}\right) \log a_{A}
$$

It is important to notice that this method can be used only if the sensor exhibits a Nernstian response towards both target and interfering ions. Therefore, it is necessary to record the full calibration curve for each ion, also for the interfering ones. An example for a $\mathrm{Li}^{+}$ISE is reported in Figure $8 \mathrm{~b}$. The calculations of the selectivity coefficients must be performed by picking the potential values falling in the concentration range in which all ions of interest show a Nernstian behaviour. Once you determine the best range for the linear fitting of each slope, you can extrapolate the potential values for $\log \left(a_{i o n}\right)=1$, and use (10) to calculate the correct selectivity coefficients. Achieving a Nernstian response also for the interfering ions is not always an easy task. If it is the case, a bias in the response will be present due to incomplete ion-exchange at the sample/ISE interface. As a result, the experimentally determined $K_{A, B}^{\text {pot }}$ values may vary remarkably. Several procedures have been proposed to tackle this issue. The simplest and most common method is based on membrane conditioning before the measurements in a solution of the least interfering ion (in our example magnesium), instead of the target one. This stratagem will stimulate a complete ion-exchange at high concentrations, resulting in unbiased values of the selectivity coefficients.

- the Separate Solution Method (SSM) II consists in the independent calibration of the sensor with respect to the ions $\mathrm{A}$ and $\mathrm{B}$. The two activities that satisfy the condition $E_{A}=E_{B}$ are used for the calculation of the selectivity coefficient according to (11).

- the Fixed Interference Method (FIM) is based on the use of solutions containing interfering ions at a constant concentration, and varying concentration of the target ion. The intersection of the extrapolated linear portions of the calibration curve plotted in a semi-logarithmic scale is used to determine the activity of the target ion $a_{A}$. In Figure 9b, an example of FIM calculations is reported for $\mathrm{a} \mathrm{Li}^{+}$ISE with respect to four interfering ions. From these values, it is possible to compute the selectivity 


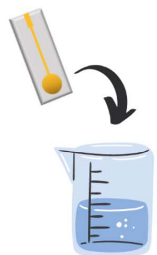

1 Conditioning in the east interfering ion solution

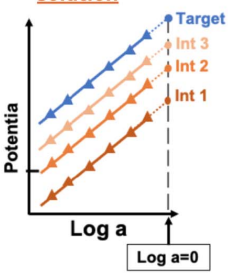

3 Calibrations with respect to other interfering ions as well target ion. Extrapolation of potentials at $\log a=0$.

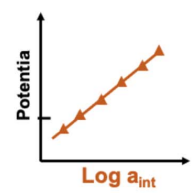

2 Calibration curve least interfering ions

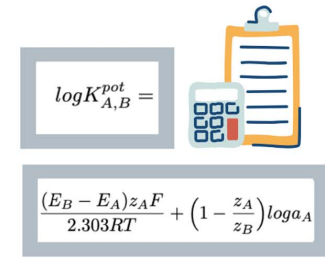

Calculation of selectivity coefficients using SSM equation.

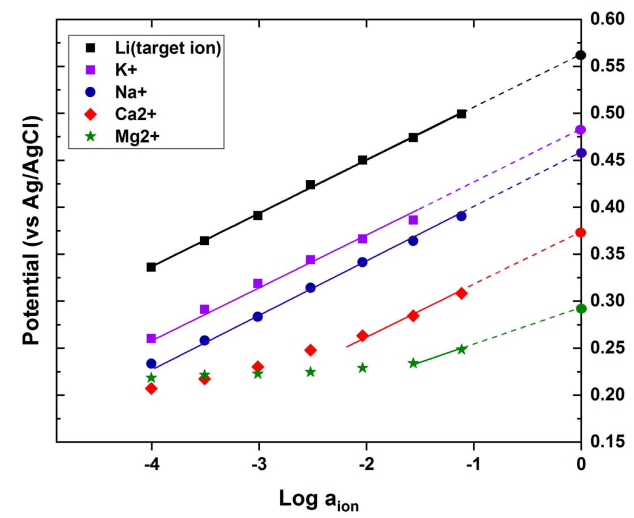

Fig. 8. a) Summary of the steps for the calculations of selectivity coefficient with the SSM. b) Example of interference measurements and calculations for a $\mathrm{Li}^{+}$ISE with the SSM.

coefficient $K_{A, B}^{p o t}$ using the following relation:

$$
K_{A, B}^{p o t}=\frac{a_{A}}{\left(a_{B}\right)^{\frac{z_{A}}{z_{B}}}},
$$

where $z_{A}$ and $z_{B}$ have the same sign.

The first and third methods described above must be considered the most reliable ones. In fact, as reported in some works [52], [53], the SSM II, also called Matched Potential Method (MPM), might give rise to negative potential changes. In this case, the selectivity coefficients obtained with this method are meaningless.

In the case of FIM, the calibration is performed with respect to the target ion, at a constant concentration of each interfering ion. On the contrary, in the SSM, the calibration must be done with respect to each interfering ion (see the difference in label between the $\mathrm{x}$-axes of Figure $8 \mathrm{~b}$ and $9 \mathrm{~b}$ ).

It is also worth to underline that the FIM can give an indication of the minimum target ion concentration at which the interference effect is not relevant. Moreover, some papers [31], [51] have shown that the simultaneous measurements of

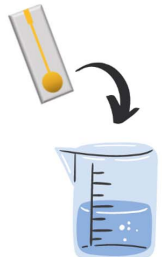

1 Conditioning in the target ion solution

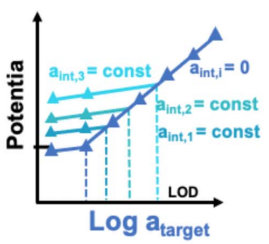

3 Repetitions of calibrations in inteferferance backgrounds

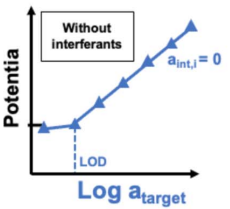

2 Calibration curve with respect to the target ion without interferants

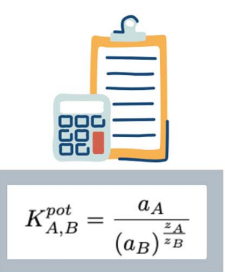

Calculation of selectivity coefficients using FIM equation.

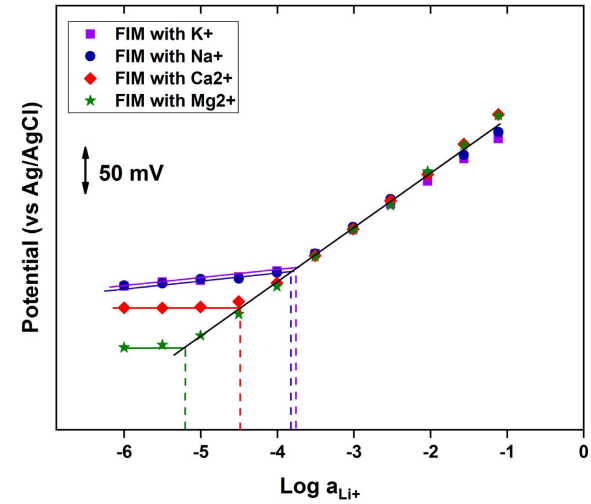

Fig. 9. a) Summary of the steps for the calculations of selectivity coefficient with the FIM. b) Example of interference measurements and calculations for $\mathrm{Li}^{+}$ISE with the FIM.

some interfering ions (in addition to target ion-sensing) can be useful to correct interference errors, thus improving the analytical accuracy.

\section{HARDWARE AND SOFTWARE INTERFACES}

Ion-sensing working principle, technology and performance evaluation criteria formerly described are concepts generally applicable to biomedical healthcare, environmental and water quality monitoring, or agriculture. In this section, more emphasis is put on biomedical applications, for which particular attention should be paid for the co-design of hardware and software interfaces in a wearable potentiometric sensing system. Namely, some guidelines are provided for a seamless integration of ion-sensors with electronic interfaces, where accurate, portable and power-efficient solutions are needed to achieve continuous and real-time on-body ion-monitoring. The main building blocks of integrated ion-sensing platforms are depicted in Figure 10, where the ISE array is interfaced to the hardware front-end performing signal acquisition, conditioning, processing, and transmission to a remote node that 


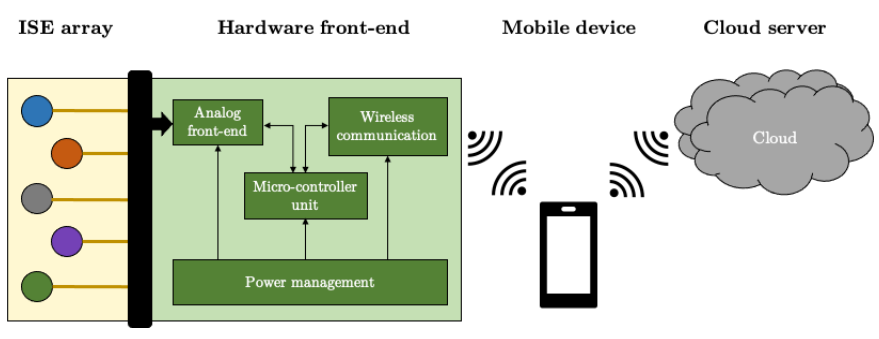

Fig. 10. Hardware and software interfaces for ion-sensors: it includes an hardware front-end for signal acquisition, conditioning, processing, and data transmission to a mobile device that is used as a visualization terminal. Data are then uploaded to cloud servers for further analysis and storage.

could be a mobile device. Data are then uploaded to cloud servers for data analysis and storage. These functional blocks are described in the following subsections.

\section{A. Hardware Front-End}

Electronic readout circuits for ion-sensors comprise an analog front-end circuitry for signal acquisition and conditioning, a processing unit, a transceiver for remote monitoring, and a power management module to supply energy to these blocks. Custom ASICs could be designed to achieve extremely small form factor front-ends, reduce power consumption, and increase Common-Mode Rejection Ratio (CMRR) [54], which are strong requirements for implantable ion-sensors. Nevertheless, the constraints from wearable potentiometric sensors are usually fulfilled by off-the-shelf integrated circuits. Namely, low-power, low-noise, high-CMRR instrumentation amplifiers are available on the market, and all components could be mounted on a Printed Circuit Board (PCB) with dimensions that are suitable for integrated ion-sensing on sweatband systems [55], [56]. Flexible PCBs are favoured for a stable sensor-skin contact since the hardware adapts to the shape of the body [7], [57]. The front-end is usually mounted on polyimide substrate such as Kapton. However, mechanical stability is a limiting factor for flexible PCBs that are dynamically bent and folded. Therefore, advanced technologies and materials should be chosen carefully in order to increase wear and electrical connection stability on such type of substrate (for instance semi-rigid PCBs, gold electrical connections). The different modules constituting the hardware front-end are described hereunder.

1) Signal Acquisition and Conditioning: Potentiometric ionsensing consists in measuring the potential difference between the ISE and the RE, in open circuit conditions. Namely, no current should flow through the electrochemical cell in order to avoid potential drops at the connector and SC interfaces. As a result, the measured OCP only depends on the potential that is built up at the sample-ISM interface when a target ion is entrapped by the membrane phase [20]. However, in practice, a polarization current of few $\mathrm{pA}$ is needed to lower the LOD, increase sensitivity and reproducibility of the sensor [6]. This quasi-zero current polarization is achieved by high impedance voltage buffers that ensure electrical isolation of the sensing platform. Moreover, the RE is tied to a virtual ground potential (analog ground or steady voltage). Attention should be paid for hybrid electrochemical platforms supporting both amperometric and potentiometric sensors. Indeed, current-sensing and voltage-sensing paths should be electrically isolated. Thus, the RE cannot be floating anymore, and the effective difference of potential between the ISE and the RE is sensed [7], [58].

Next, a differential amplifier is needed to resolve the OCP of the two-electrodes electrochemical cell. This reduces the common-mode noise as well. A gain stage of four or five is set so as to leverage the full dynamic range of the Analog-to-Digital Converter (ADC) downstream [59]. This allows increasing OCP readout resolution. Both buffering and differential amplification could be implemented with an offthe-shelf instrumentation amplifier. Low-power, high-CMRR, rail-to-rail instrumentation amplifiers are available on the market [60].

Besides, the signal conditioning path includes a low-pass filter serving as anti-aliasing element before sampling the signal, and it is used to remove high-frequency noise and interference from OCP measurements as well. A unity-gain low-pass filter is typically implemented with a Sallen-Key topology that could be cascaded to form high-order filters. A 4th-order SallenKey Butterworth low-pass filter is a common design choice to achieve a flat pass-band response and a steep $80 \mathrm{~dB} /$ decade roll-off. Sub-Hz corner frequency are implemented since the potentiometric sensor signals are DC potentials [7], [59].

Eventually, the output signal is sampled by a calibrated ADC. The in-built ADC from a Micro-Controller Unit (MCU) is generally used if it complies with accuracy requirements. An alternative to the use of a differential amplifier is to sense the potential of ISE and RE directly, with positive and negative input of the ADC, respectively [61]. Furthermore, oversampling and averaging are implemented in software on the MCU so as to improve resolution and accuracy of OCP readout.

2) Wireless Data Transmission: The elaborated data is sent to a transceiver, leveraging serial communication capabilities of the on-board MCU -Universal Asynchronous Receiver Transmitter port, Serial Peripheral Interface, Inter-Integrated Circuit bus. Then, the wireless module transmits the data to a mobile device for visualization. Several wireless communication protocols are available: Bluetooth low energy, nearfield communication, ZigBee, ANT+, Wi-Fi, and many others. Its choice depends on the specifications from the ion-sensing application and some criteria such as the range of transmission, the bit rate, the number of channels supported and channel bandwidth, the power-saving schemes, and the protocol efficiency for processing the data packets to convey [62]. These figures are considered, targeting a power-efficient, continuous, and real-time ion-monitoring.

3) Power Management: Reducing power consumption is a salient priority in the design of wearable devices since they need to operate continuously for a large time span. It is even more intricate for multi-sensing platforms collecting signals from array of sensors. Rechargeable flat lithium-ion batteries with nominal voltage of $3.7 \mathrm{~V}$ are a common solution to power-up the hardware front-end. The choice of the battery capacity is conditioned by its form factor that should enable its integration into a portable system. Coin cell batteries are 
another alternative, but these batteries are rather heavy and hardly compatible with flexible sensing platforms.

Voltage-boost regulators, DC-DC converters, or LowDropout regulators are used in order to get stable and regulated voltage supplies for the analog front-end, the MCU, and the wireless module. Suitable protection circuitry are also added to protect the battery from unwanted output shorts and overcharging.

4) Data Visualization and Storage: Custom mobile applications are designed to provide an user-friendly interface enabling the visualization of the biological information of interest in real-time. Device pairing and secure connection, configuration of the wireless transmission process (data rate, potentiometric channel to acquire data from), plotting, and sharing features are desired services to have on the application. The small memory resources of the mobile device suggest to upload the aggregated biological information to cloud servers, where they could be stored and further analysed by experts or through complex data analytics.

\section{B. Chemometric Tools for Ion-Sensors}

Chemometrics is an interdisciplinary field that leverages signal processing, mathematical methods, and statistics, to address problems in analytical chemistry, biochemistry, or medicine [63]. A data-driven approach is implemented where the objective is to extract relevant information from sensor responses in order to solve descriptive problems (understanding the properties of chemical systems), or for predictive tasks (predict attributes of components in chemical systems). Within a multi-ion-sensing framework, chemometric tools are developed to get an accurate estimate of the electrolytes of interest in the sample. For biomedical applications, it means providing meaningful feedback to the user about its physiological status, hydration state, or muscle fatigue, from the signals output by ISEs. For water quality monitoring, it consists in measuring the concentration of multiple free ions and pollutants to ensure that the water complies with quality standards. The assessment of unknown samples is possible after thorough in-the-field calibration procedures, that are mapping the sensor responses to conclusive physiological information or water quality compliance, for the two previous examples. The massive amount of data collected from several subjects or samples could be used as a database for data mining tools and machine learning models that extract relevant analytical information from these aggregated data. The coupling of these chemometric software tools with a multi-ion-sensing platform forms an electronic tongue system that is constructed to improve the prediction accuracy of the electrolytes of interest [64]. In addition, being able to warn the user when one electrolyte is out of its nominal concentration range, or trend, is a desired feature for such intelligent sensor.

An important challenge in accurate ion-sensing arises from ion interference, as explained in Section III-D. Interference comes from the sample matrix that inherently contains background electrolytes, or from extrinsic compounds (e.g. toxic compounds and trace metals). Ion interference significantly distorts sensor response and increases the lower LOD of the sensor. This is critical if the non-linearity affects the range of interest of the ions to be tracked. A typical approach is to use a multi-ion-sensing platform that monitors the activity of the target ions and also the main interfering electrolytes, so that the latter information could be used as an additional feature for the machine learning model in a feedback network. Artificial neural networks are commonly built to optimize this multivariate calibration procedure [65], [66].

The machine learning models are implemented in cloud servers to leverage computational and memory resources. However, latency could be a bottleneck for real-time ionmonitoring. Thus, models with a lower computational complexity, or pre-trained models could be deployed onto edge devices at the vicinity of the sensors, e.g. Raspberry-Pi, Field Programmable Gate Array, Arduino boards, in order to get a coarse estimation of the parameters of interest. The power consumption of the overall internet-of-things system is a predominant factor for the design choices and trade-offs.

\section{Conclusion and Future Perspectives}

The interests in the improvement of the all-solid-state ionsensing technology has grown rapidly in the last years as a consequence of the increasing demand for remote and wearable ion-sensors. This typically exploit a variety of novel solid-contact materials to enhance performance.

These devices have apparently a simple working principle. However, the theory and characterization practice of these class of sensors differs in some cases from traditional definitions. In this tutorial, we have discussed the proper protocols required to achieve reproducible results and especially to define sensing parameters that can be safely compared to literature ones. A brief overview of the major hardware and software design considerations is also given to provide a complete overview of the technology.

Despite the fast advancement of this technology in recent years in several fields, there is still large room for improvements to fabricate efficient all-solid-state ISEs for portable and wearable devices suitable for commercialization. Certainly, the recent expansion of the interests on ISEs will contribute to the fast growth of the technology, with significant impact on various fields of research, like healthcare and water quality monitoring.

\section{REFERENCES}

[1] Y. Lyu et al., "Solid-contact ion-selective electrodes: Response mechanisms, transducer materials and wearable sensors," Membranes, vol. 10, no. 6, pp. 1-24, 2020.

[2] Y. Shao, Y. Ying, and J. Ping, "Recent advances in solid-contact ionselective electrodes: Functional materials, transduction mechanisms, and development trends," Chem. Soc. Rev., vol. 49, no. 13, pp. 4405-4465, 2020.

[3] A. Mena-Bravo and M. D. L. de Castro, "Sweat: A sample with limited present applications and promising future in metabolomics," J. Pharmaceutical Biomed. Anal., vol. 90, pp. 139-147, Mar. 2014, doi: 10.1016/j.jpba.2013.10.048.

[4] G. Matzeu, L. Florea, and D. Diamond, "Advances in wearable chemical sensor design for monitoring biological fluids," Sens. Actuators B, Chem., vol. 211, pp. 403-418, May 2015.

[5] A. J. Bandodkar, I. Jeerapan, and J. Wang, "Wearable chemical sensors: Present challenges and future prospects," ACS Sensors, vol. 1, no. 5, pp. 464-482, May 2016.

[6] E. Lindner and R. E. Gyurcsányi, "Quality control criteria for solidcontact, solvent polymeric membrane ion-selective electrodes," J. Solid State Electrochem., vol. 13, no. 1, pp. 51-68, Jan. 2009. 
[7] W. Gao et al., "Fully integrated wearable sensor arrays for multiplexed in situ perspiration analysis," Nature, vol. 529, pp. 509-514, Jan. 2016.

[8] S. Anastasova-Ivanova et al., "A wearable multisensing patch for continuous sweat monitoring," Biosens. Bioelectron., vol. 93, p. 730, May 2017

[9] V. A. T. Dam, M. A. G. Zevenbergen, and R. van Schaijk, "Flexible chloride sensor for sweat analysis," Procedia Eng., vol. 120, pp. 237-240, Jan. 2015.

[10] F. Criscuolo, F. Cantu, I. Taurino, S. Carrara, and G. De Micheli, "A wearable electrochemical sensing system for non-invasive monitoring of lithium drug in bipolar disorder," IEEE Sensors J., vol. 21, no. 8 , pp. 9649-9656, Apr. 2021.

[11] S. T. Keene, D. Fogarty, R. Cooke, C. D. Casadevall, A. Salleo, and O. Parlak, "Wearable organic electrochemical transistor patch for multiplexed sensing of calcium and ammonium ions from human perspiration," Adv. Healthcare Mater, vol. 8, no. 24, pp. 1-8, 2019.

[12] F. Criscuolo, I. Taurino, V. A. Dam, and F. Catthoor, "Fast procedures for the electrodeposition of platinum nanostructures on miniaturized electrodes for," Sensors, vol. 19, no. 2260, pp. 1-12, 2019.

[13] S. Anastasova et al., "Disposable solid-contact ion-selective electrodes for environmental monitoring of lead with ppb limit-ofdetection," Electrochimica Acta, vol. 73, pp. 93-97, Jul. 2012, doi: 10.1016/j.electacta.2011.10.089

[14] J. C. Chou, M. W. Su, C. C. Chen, and S. Y. Yang, " $\mathrm{Cl}^{-}$and $\mathrm{H}^{+}$sensing devices for water quality monitoring system," in Proc. IEEE Int. Symp. Circuits Syst. (ISCAS), May 2012, pp. 2043-2046.

[15] R. Athavale, C. Dinkel, B. Wehrli, E. Bakker, G. A. Crespo, and A. Brand, "Robust solid-contact ion selective electrodes for highresolution in situ measurements in fresh water systems," Environ. Sci. Technol. Lett., vol. 4, no. 7, pp. 286-291, Jul. 2017.

[16] M. A. Zayed, A. A. Abbas, W. H. Mahmoud, A. E. Ali, and G. G. Mohamed, "Development and surface characterization of a bis(aminotriazoles) derivative based renewable carbon paste electrode for selective potentiometric determination of $\mathrm{Cr}$ (III) ion in real water samples," Microchem. J., vol. 159, Dec. 2020, Art. no. 105478, doi: 10.1016/j.microc.2020.105478.

[17] G. A. Crespo, "Recent Advances in ion-selective membrane electrodes for in situ environmental water analysis," Electrochimica Acta, vol. 245, pp. 1023-1034, Aug. 2017, doi: 10.1016/j.electacta.2017.05.159.

[18] S. Chowdhury, B. Rooj, A. Dutta, and U. Mandal, "Review on recent advances in metal ions sensing using different fluorescent probes," J. Fluorescence, vol. 28, no. 4, pp. 999-1021, Jul. 2018.

[19] I. Fakih et al., "Selective ion sensing with high resolution large area graphene field effect transistor arrays," Nature Commun., vol. 11, no. 1, pp. 1-12, Dec. 2020, doi: 10.1038/s41467-020-16979-y.

[20] J. Hu, A. Stein, and P. Bühlmann, "Rational design of all-solid-state ion-selective electrodes and reference electrodes," Trends Anal. Chem., vol. 76, pp. 102-114, Feb. 2016

[21] N. Rubinova, K. Chumbimuni-Torres, and E. Bakker, "Solid-contact potentiometric polymer membrane microelectrodes for the detection of silver ions at the femtomole level," Sens. Actuators B, vol. 121, no. 1, pp. 213-223, 2006

[22] E. Bakker, P. Bühlmann, and E. Pretsch, "Polymer membrane ionselective electrodes \pm what are the limits?" Electroanalysis, vol. 11, no. 13, pp. 915-933, Sep. 1999.

[23] C. C. Rundle, "A beginners guide to ion-selective electrode measurements," Nico2000 Ltd, London, U.K., Tech. Rep., Jan. 2011, pp. 1-30.

[24] P. Kumar, K.-H. Kim, V. Bansal, T. Lazarides, and N. Kumar, "Progress in the sensing techniques for heavy metal ions using nanomaterials," J. Ind. Eng. Chem., vol. 54, pp. 30-43, Oct. 2017, doi: 10.1016/j.jiec.2017.06.010.

[25] B. Kaur, N. Kaur, and S. Kumar, "Colorimetric metal ion sensors-A comprehensive review of the years 2011-2016," Coordination Chem. Rev., vol. 358, pp. 13-69, Mar. 2018, doi: 10.1016/j.ccr.2017.12.002.

[26] N. Kaur and S. Kumar, "Colorimetric metal ion sensors," Tetrahedron, vol. 67, no. 67, pp. 9233-9264, 2011, doi: 10.1016/j.tet.2011.09.003.

[27] W. E. Morf, The Principles of Ion-Selective Electrodes and of Membrane Transport, vol. 2. Amsterdam, The Netherlands: Elsevier, 1981.

[28] P. C. Meier, "Two-parameter Debye-Hückel approximation for the evaluation of mean activity coefficients of 109 electrolytes," Analytica Chimica Acta, vol. 136, pp. 363-368, Jan. 1982.

[29] I. Taurino, G. Sanzo, F. Mazzei, G. Favero, G. De Micheli, and S. Carrara, "Fast synthesis of platinum nanopetals and nanospheres for highly-sensitive non-enzymatic detection of glucose and selective sensing of ions," Sci. Rep., vol. 5, p. 15277, Oct. 2015.

[30] J. Bobacka, A. Ivaska, and A. Lewenstam, "Potentiometric ion sensors," Chem. Rev., vol. 108, no. 2, pp. 329-351, Jan. 2008.
[31] A. Lewenstam, "Routines and challenges in clinical application of electrochemical ion-sensors," Electroanalysis, vol. 26, no. 6, pp. 1171-1181, Jun. 2014

[32] H. Hirata, "Copper(I) sulphide-impregnated silicone rubber membranes as selective electrodes for copper(II) ions," Talanta, vol. 17, no. 9 , pp. 2-6, 1970.

[33] R. W. Cattrall, H. Freiser, and R. W. Cattrall, "Coated wire ion-selective electrodes," Anal. Chem., vol. 43, no. 13, pp. 1905-1906, 1971.

[34] E. Lindner and Y. Umezawa, "Performance evaluation criteria for preparation and measurement of macro- and microfabricated ion-selective electrodes (IUPAC technical report)," Pure Appl. Chem., vol. 80, no. 1, pp. 85-104, Jan. 2008.

[35] J. Bobacka, "Conducting polymer-based solid-state ion-selective electrodes," Electroanalysis, vol. 18, no. 1, pp. 7-18, 2006.

[36] T. Yin and W. Qin, "Applications of nanomaterials in potentiometric sensors," Trends Anal. Chem., vol. 51, pp. 79-86, Nov. 2013.

[37] A. Cadogan, Z. Gao, A. Lewenstam, A. Ivaska, and D. Diamond, "Allsolid-state sodium-selective electrode based on a calixarene ionophore in a poly(vinyl chloride) membrane with a polypyrrole solid contact," Anal. Chem., vol. 64, no. 21, pp. 2496-2501, Nov. 1992

[38] J. Bobacka, T. Lindfors, M. McCarrick, A. Ivaska, and A. Lewenstam, "Single-piece all-solid-state ion-selective electrode," Anal. Chem., vol. 67, no. 20, pp. 3819-3823, Oct. 1995.

[39] J. Bobacka, M. Mccarrick, and A. Ivaskat, "All solid-state poly (vinyl chloride) membrane ion-selective electrodes with poly (3-octylthiophene) solid internal contact," Analyst, vol. 119, no. 9, pp. 1985-1991, 1994.

[40] J. Bobacka, "Potential stability of all-solid-state ion-selective electrodes using conducting polymers as ion-to-electron transducers," Anal. Chem., vol. 71, no. 21, pp. 4932-4937, Oct. 1999.

[41] C.-Z. Lai, M. A. Fierke, A. Stein, and P. Bühlmann, "Ion-selective electrodes with three-dimensionally ordered macroporous carbon as the solid contact," Anal. Chem., vol. 79, no. 12, pp. 4621-4626, Jun. 2007.

[42] G. A. Crespo, S. Macho, and F. X. Rius, "Ion-selective electrodes using carbon nanotubes as ion-to-electron transducers," Anal. Chem., vol. 80, no. 4, pp. 1316-1322, Feb. 2008.

[43] J. Ping, Y. Wang, J. Wu, and Y. Ying, "Development of an all-solid-state potassium ion-selective electrode using graphene as the solid-contact transducer," Electrochem. Commun., vol. 13, no. 12, pp. 1529-1532, Dec. 2011.

[44] F. Li et al., "All-solid-state potassium-selective electrode using graphene as the solid contact," Analyst, vol. 137, no. 3, pp. 618-623, 2012.

[45] E. Jaworska, M. Wójcik, A. Kisiel, J. Mieczkowski, and A. Michalska, "Gold nanoparticles solid contact for ion-selective electrodes of highly stable potential readings," Talanta, vol. 85, no. 4, pp. 1986-1989, Sep. 2011

[46] B. Paczosa-Bator, L. Cabaj, R. Piech, and K. Skupień, "Potentiometric sensors with carbon black supporting platinum nanoparticles," Anal. Chem., vol. 85, no. 21, pp. 10255-10261, Nov. 2013

[47] T. Yin, D. Pan, and W. Qin, "All-solid-state polymeric membrane ionselective miniaturized electrodes based on a nanoporous gold film as solid contact," Anal. Chem., vol. 86, pp. 11038-11044, Nov. 2014.

[48] F. Criscuolo, I. Taurino, F. Stradolini, S. Carrara, and G. De Micheli, "Highly-stable $\mathrm{Li}^{+}$ion-selective electrodes based on noble metal nanostructured layers as solid-contacts," Analytica Chimica Acta, vol. 1027, pp. 22-32, Oct. 2018.

[49] E. Bakker and E. Pretsch, "Potentiometric sensors for trace-level analysis," Trends Anal. Chem., vol. 24, no. 3, pp. 199-207, 2005.

[50] M. Guzinski, J. M. Jarvis, B. D. Pendley, and E. Lindner, "Equilibration time of solid contact ion-selective electrodes," Anal. Chem., vol. 87, no. 13, pp. 6654-6659, Jul. 2015.

[51] R. P. Buck and E. Lindner, "Recomendations for nomenclature of ion-selective electrodes (IUPAC recommendations 1994)," Pure Appl. Chem., vol. 66, no. 12, pp. 2527-2536, 1994.

[52] E. Bakker, E. Pretsch, and P. Bühlmann, "Selectivity of potentiometric ion sensors," Anal. Chem., vol. 72, pp. 1127-1133, Mar. 2000.

[53] M. A. Piñeros, J. E. Shaff, L. V. Kochian, and E. Bakker, "Selectivity of liquid membrane cadmium microelectrodes based on the ionophore N,N,N0,N0-tetrabutyl-3,6-dioxaoctanedithioamide," Electroanalysis, vol. 10, no. 14, pp. 937-941, Oct. 1998.

[54] W.-S. Wang et al., "Real-time telemetry system for amperometric and potentiometric electrochemical sensors," Sensors, vol. 11, no. 9, pp. 8593-8610, Sep. 2011.

[55] A. J. Bandodkar et al., "Epidermal tattoo potentiometric sodium sensors with wireless signal transduction for continuous non-invasive sweat monitoring," Biosensors Bioelectron., vol. 54, pp. 603-609, Apr. 2014, doi: 10.1016/j.bios.2013.11.039. 
[56] M. Bariya, H. Y. Y. Nyein, and A. Javey, "Wearable sweat sensors," Nat. Electron., vol. 1, no. 3, pp. 160-171, 2018.

[57] I. N. Hanitra et al., "A flexible front-end for wearable electrochemical sensing," in Proc. IEEE Int. Symp. Med. Meas. Appl. (MeMeA), Jun. 2018, pp. 558-563.

[58] I. N. Hanitra, F. Criscuolo, N. Pankratova, S. Carrara, and G. D. Micheli, "Multichannel front-end for electrochemical sensing of metabolites, drugs, and electrolytes," IEEE Sensors J., vol. 20, no. 7, pp. 3636-3645, Apr. 2020.

[59] I. N. Hanitra, F. Criscuolo, S. Carrara, and G. De Micheli, "Multi-target electrolyte sensing front-end for wearable physical monitoring," in Proc. 15th Conf. Ph. D Res. Microelectron. Electron. (PRIME), Jul. 2019, pp. $249-252$.

[60] S. Emaminejad et al., "Autonomous sweat extraction and analysis applied to cystic fibrosis and glucose monitoring using a fully integrated wearable platform," Proc. Nat. Acad. Sci. USA, vol. 114, no. 18, pp. 4625-4630, 2017.

[61] H. Y. Y. Nyein et al., "A wearable microfluidic sensing patch for dynamic sweat secretion analysis," ACS Sensors, vol. 3, no. 5, pp. 944-952, 2018.

[62] P. Kassal, M. D. Steinberg, and I. M. Steinberg, "Wireless chemical sensors and biosensors: A review," Sens. Actuators B, Chem., vol. 266, pp. 228-245, Aug. 2018.

[63] S. Wold, "Chemometrics; what do we mean with it, and what do we want from it?" Chemometric Intell. Lab. Syst., vol. 30, no. 1, pp. 109-115, Nov. 1995.

[64] Y. Vlasov, A. Legin, A. Rudnitskaya, C. Di Natale, and A. D’Amico, "Nonspecific sensor arrays ('electronic tongue') for chemical analysis of liquids (IUPAC technical report)," Pure Appl. Chem., vol. 77, no. 11, pp. 1965-1983, 2005.

[65] L. Wang, Y. Cheng, D. Lamb, M. Megharaj, and R. Naidu, "Application of ion selective electrode array to simultaneously determinate multifree ions in solution," Environ. Technol. Innov., vol. 15, Aug. 2019, Art. no. 100424, doi: 10.1016/j.eti.2019.100424.

[66] L. Wang et al., "Simultaneously determining multi-metal ions using an ion selective electrode array system," Environ. Technol. Innov., vol. 6, pp. 165-176, Nov. 2016.

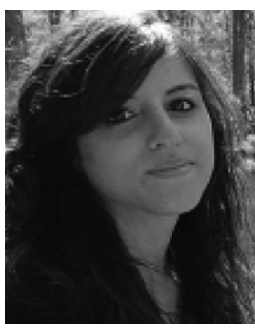

Francesca Criscuolo received the B.Sc. and M.Sc. (cum laude) degrees in materials engineering and nanotechnology from the Politecnico di Milano, Italy, and the Ph.D. degree from the Integrated System Laboratory, EPFL, Lausanne, Switzerland, in April 2020, with her thesis "Wearable multi-electrode platform for ion-sensing." During her studies, she participated in several exchange programs in France, Czech Republic, The Netherlands, and Belgium. She spent one year at TU Delft, where she specialized in bionanomaterials and in nanoelectronics. In 2015, she worked with the Energy Storage Group, IMEC, Leuven, Belgium. She is currently a Postdoctoral Fellow at EPFL, where she focuses on novel electrochemical biosensors for healthcare and diagnostics.

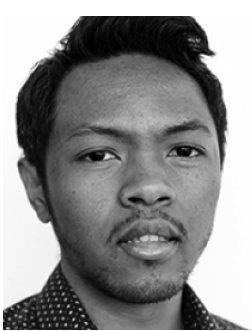

Mandresy Ivan Ny Hanitra (Member, IEEE) received the B.S. degree in physics and chemistry from the Grenoble Institute of Technology, Grenoble, France, in 2014, the joint M.S. degree in micro and nanotechnologies for integrated systems between the Politecnico di Torino, Turin, Italy; the Grenoble Institute of Technology, and EPFL, Lausanne, Switzerland, in 2016. $\mathrm{He}$ is currently pursuing the Ph.D. degree in microelectronics and microsystems. He pursued his master's thesis at the Electronics Laboratory (Prof. Kayal, EPFL), developing low-power systems for photoplethysmographic-based heart rate monitoring. He joined the Integrated Systems Laboratory (Prof. De Micheli, EPFL) in October 2016, where he designed and realized multi-target electrochemical sensing platforms. His research involves the co-design and development of electronic interfaces for electrochemical sensors. A strong emphasis of his research is laid on developing efficient data processing tools and optimization algorithms within a multi-sensing paradigm. His work is aiming at physical status monitoring and advanced healthcare diagnosis for wearable applications.

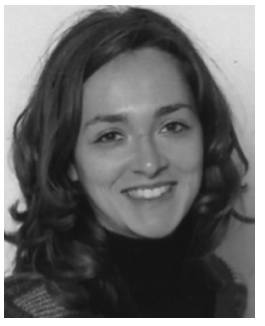

Irene Taurino received the B.Sc. degree in biomedical engineering from the Politecnico di Torino, the dual M.Sc. (summa cum laude) degree in biomedical engineering from the Politecnico di Milano, and the Ph.D. degree in microsystems and microelectronics from EPFL in 2015. She was a Visiting Student with the Khademhosseini Laboratory, Harvard-MIT Division of Health Sciences and Technology, Cambridge, MA, USA, for the integration of an electrochemical device in a fluidic system for the continuous control of bioreactors containing human tissue constructs. She has three years of industrial experience in research and development working on devices for respiratory drug delivery and aerosol characterization methods. She is working on nanotechnology and electrochemical biosensors with EPFL.

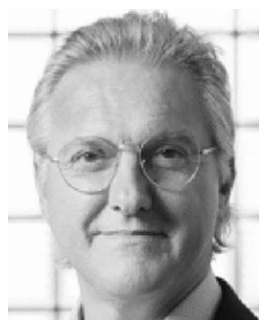

Sandro Carrara (Fellow, IEEE) is currently a Faculty with EPFL, Lausanne, Switzerland, and a former Professor with the University of Genoa, Italy, and the University of Bologna, Italy. Along his carrier, he published seven books, with prestigious publishers like Springer/Nature and Cambridge University Press. He published more than 270 articles and holds 14 patents. He was a member of the BoG of the IEEE CASS and the IEEE Sensors Council. He was a recipient of the IEEE Sensors Council Technical Achievement Award. $\mathrm{He}$ is the Editor-in-Chief of the IEEE SENSORS JOURNAL and an Associate Editor of IEEE TRANSACTIONS ON BIOMEDICAL CIRCUITS AND SYSTEMS.

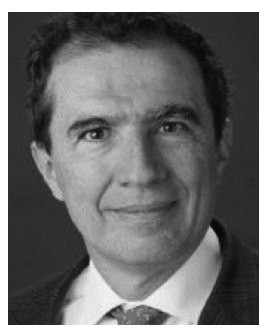

Giovanni De Micheli (Life Fellow, IEEE) is a Professor and the Director of the Integrated Systems Laboratory, EPFL, Lausanne, Switzerland. Previously, he was a Professor of Electrical Engineering at Stanford University. He was the Director of the Electrical Engineering Institute (IEL), EPFL, from 2008 to 2019, and the Program Leader of the Swiss Federal Nano-Tera.ch Program. His current research interests include several aspects of design technologies for integrated circuits and systems, such as synthesis for emerging technologies. $\mathrm{He}$ is also interested in heterogeneous platform design, including electrical components and biosensors, as well as in data processing of biomedical information. Prof. De Micheli is a Fellow of $\mathrm{ACM}$, a member of the Academia Europaea, and an International Honorary Member of the American Academy of Arts and Sciences. He was a recipient of the 2020 IEEE/CEDA Richard Newton Technical Impact Award, the 2019 ACM/SIGDA Pioneering Achievement Award, and the 2016 EDAA Lifetime Achievement Award. 